\title{
Diagnosis de la defectología que de forma accidental suele presentar la laminación de tubería de altas prestaciones ${ }^{(\bullet)}$
}

\author{
S. Gutiérrez de Sáiz-Solabarría ${ }^{(*)}$ \\ Resumen Se estudian las posibles causas que dan lugar a la defectología que, de forma accidental, suele pre- \\ sentarse en la laminación de tubería de altas prestaciones. En el presente trabajo se contemplan las \\ calidades de acero de la norma API (American Petroleum Institute) en su especificación 5CT (1) para \\ los grados C75-1 (acero al C-Mn aleado con Mo) y J55 (acero al C-Mn) y en su especificación 5L (2) \\ para el grado A. Se caracteriza la tubería tanto química como metalúrgicamente, se diagnostican los \\ defectos y se establecen conclusiones. \\ Palabras clave: Defectología en la laminación. Tubería de altas prestaciones, Calidades C75.1, \\ J55 y Gr A. Normas API 5CT y 5L.
}

\section{Diagnosis of the defectology which usually arises when rolling high-performing pipes}

\begin{abstract}
The possible causes are studied which produce the defectology that, incidentally, is usually present at high-performing pipe rolling processes. The steel qualities included in Specification 5CT (1) for grade C75-1 (C-Mn-Mo-alloyed steel) and grade J55 (C-Mn steel) as well as those qualities regarding Specification 5L (2) for grade A of Standard API (American Petroleum Institute) are studied herein. Both chemical and metallurgical characterizations of the pipe are provided. Defects are diagnosed and conclusions are set.
\end{abstract}

Keywords: Defectology at the rolling-process. High-performing pipe, Qualities C75.1, J55 and Gr A. Standards API 5CT and 5L.

\section{INTRODUCCIÓN}

En la fabricación de tubería para altas prestaciones (3) se suelen producir de forma accidental unos tipos de defectos que, en todos los casos, deben ser estudiados, diagnosticados y evaluados. Estos defectos tienen su origen, unas veces, en el lingote

$(\bullet)$ Trabajo recibido el día 17 de febrero de 1994.

$\left.{ }^{*}\right)$ Dpto. Laboratorios. Unidad I+D. Babcock \& Wilcox Española, S.A. Apdo. 294. 48080-Bilbao (España).

Dpto. Ciencia de los Materiales e Ingeniería Metalúrgica. UPV/EHU. Apdo. 644. 48080-Bilbao (España). de partida, otras, se producen en los trenes de laminación y, las menos, en el tratamiento térmico final (4). Bien es cierto que a veces no es un único proceso el causante del defecto sino que es la actuación sinérgica de los tres procesos comentados. Normalmente los defectos, una vez diagnosticados, se evalúan con respecto a las normas, códigos y especificaciones técnicas al efecto. En algunos casos, los menos, la singularidad del defecto implica reconsideración en el cálculo y, en otros, se considera fundamental y determinante el criterio del metalurgista (5). La diagnosis y evaluación permitirán, por tanto, decidir si la tubería es adecuada para el uso que se contempla. 


\section{ALCANCE DEL ESTUDIO}

En el presente trabajo se estudia y diagnostica la defectología que presentan cuatro tubos diferentes fabricados según la norma API (1 y 2). Dos de ellos de un diámetro exterior de $73 \mathrm{~mm}$ y calidad C75.1 (C-Mn aleado con Mo), un tubo de diámetro exterior igual a $60 \mathrm{~mm}$ y calidad J55 (C-Mn), y el tubo restante de $48 \mathrm{~mm}$ de diámetro exterior y calidad Gr A.

\section{OBJETIVO}

El objetivo de este trabajo es diagnosticar la diferente defectología que presentan cuatro tubos fabricados según la norma API en sus calidades C75.1, J55 y Gr A, respectivamente.

\section{PARTE EXPERIMENTAL}

Para la realización de los ensayos se obtuvieron diferentes muestras representativas de la defectología observada y se procedió a su caracterización.

\subsection{Caracterización química}

La caracterización química se realizó utilizando las técnicas instrumentales de espectrometría de emisión atómica por descarga eléctrica (AES) y por acoplamiento de plasma inductivo (ICP), obteniéndose los datos que presenta la tabla I, en la que se observa que se cumplen las calidades C75.1, J55 y $\mathrm{Gr}$ A especificadas. El contenido de elementos residuales se considera normal.

\subsection{Ensayos de dureza}

Para los ensayos de dureza se utilizó un esclerómetro Rockwell en sus escalas B y C, obteniéndose los resultados que muestra la tabla II, donde se observa que se cumplen las calidades C75.1, J55 y Gr A especificadas.

\subsection{Caracterización metalográfica}

La caracterización metalográfica se llevó a cabo mediante microscopía óptica, incorporando técnicas de polarización e interferencia así como ilumina ción por iodo-cuarzo y vapor de mercurio, obte-

Tabla I. - Composición de la tubería estudiada

TABLE I.- Chemical composition of the studied pipe

\begin{tabular}{|c|c|c|c|c|c|c|c|c|c|c|c|c|}
\hline \multicolumn{1}{|c|}{ Tubo } & \multicolumn{10}{|c|}{ Composición, \% en masa } \\
\hline Núm. & Diám., mm & C & Mn & Si & P & S & Mo & Cr & Ni & Cu & Al & Sn \\
\hline 1 & 73 & 0,38 & 1,41 & 0,23 & 0,021 & 0,010 & 0,16 & 0,08 & 0,10 & 0,21 & 0,015 & 0,015 \\
2 & 73 & 0,37 & 1,47 & 0,22 & 0,023 & 0,007 & 0,17 & 0,11 & 0,11 & 0,23 & 0,006 & 0,018 \\
3 & 60 & 0,27 & 1,22 & 0,21 & 0,015 & 0,006 & - & 0,04 & 0,06 & 0,14 & 0,018 & 0,013 \\
4 & 48 & 0,12 & 0,52 & 0,24 & 0,018 & 0,007 & 0,03 & 0,09 & 0,11 & 0,22 & 0,032 & 0,016 \\
\hline
\end{tabular}

TABLA II.- Dureza y su equivalencia en resistencia TABLE II.- Hardness values and corresponding strength equivalences

\begin{tabular}{|c|c|c|c|c|}
\hline \multicolumn{2}{|c|}{} & \multicolumn{2}{c|}{$\begin{array}{c}\text { Dureza } \\
\text { Rockwell }\end{array}$} & $\begin{array}{c}\text { Equivalencia } \\
\text { en resistencia }\end{array}$ \\
\hline Núm. & Diám., mm & HRB & HRC & $\mathrm{kg} / \mathrm{mm}^{2}$ \\
\hline 1 & 73 & - & $21-22$ & $81 \pm 1$ \\
2 & 73 & - & $15 \pm 1$ & $70 \pm 1$ \\
3 & 60 & $88-89$ & - & $62 \pm 1$ \\
4 & 48 & $69-70$ & - & $43-44$ \\
\hline
\end{tabular}

niéndose, respectivamente, los valores que se muestran en las tablas III y IV.

\subsubsection{Impresión Baumann}

La impresión Baumann se realizó en una de las secciones transversales de cada tubo, observándose que el azufre se encuentra uniformemente distribuido, es decir, que el acero, en todos los casos, se encuentra bien calmado, según se deduce de las impresiones Baumann que se muestran en la figura 9, en la que, además, se pueden observar los diámetros y espesores de la tubería estudiada. 
TABLA III.-Estructuras de la tubería estudiada

TABLE III.- Structures of the investigated pipe

\begin{tabular}{|c|c|c|c|}
\hline \multicolumn{2}{|c|}{ Tubo } & \multirow{2}{*}{ Estructura } & \multirow{2}{*}{ Figura } \\
\hline Núm. & $\begin{array}{c}\text { Diám., } \\
\text { mm }\end{array}$ & & \\
\hline $\begin{array}{l}1 \\
2\end{array}$ & $\begin{array}{l}73 \\
73\end{array}$ & $\begin{array}{l}\text { Estructuras intermedias } \\
\text { de temple } \\
\text { Matriz de grano fino }\end{array}$ & 1 y 2 \\
\hline 3 & 60 & $\begin{array}{l}\text { Distribución uniforme } \\
\text { del constituyente } \\
\text { perlítico en una matriz } \\
\text { ferrítica de grano fino } \\
\text { (núms. } 9 \text { - } 91 / 2 \text { de ASTM). } \\
\text { Orientación en bandas }\end{array}$ & 3 \\
\hline 4 & 48 & $\begin{array}{l}\text { Distribución uniforme } \\
\text { del constituyente } \\
\text { perlítico en una matriz } \\
\text { ferrítica de grano fino } \\
\text { (núms. } 8 \text { - } 81 / 2 \text { de ASTM) }\end{array}$ & 4 \\
\hline
\end{tabular}

\subsection{Defectología}

A partir de la observación visual con lupa estereoscópica, macroscopía y microscopía óptica, se obtuvieron los resultados que se ofrecen en los siguientes subapartados:

\subsubsection{Tubos núms. 1 y 2: Calidad C75.1 y diámetro $73 \mathrm{~mm}$}

En los tubos núms. 1 y 2 , la superficie exterior presentaba fisuras y grietas finas, cortas y paralelas entre sí, de localización transversal con respecto a

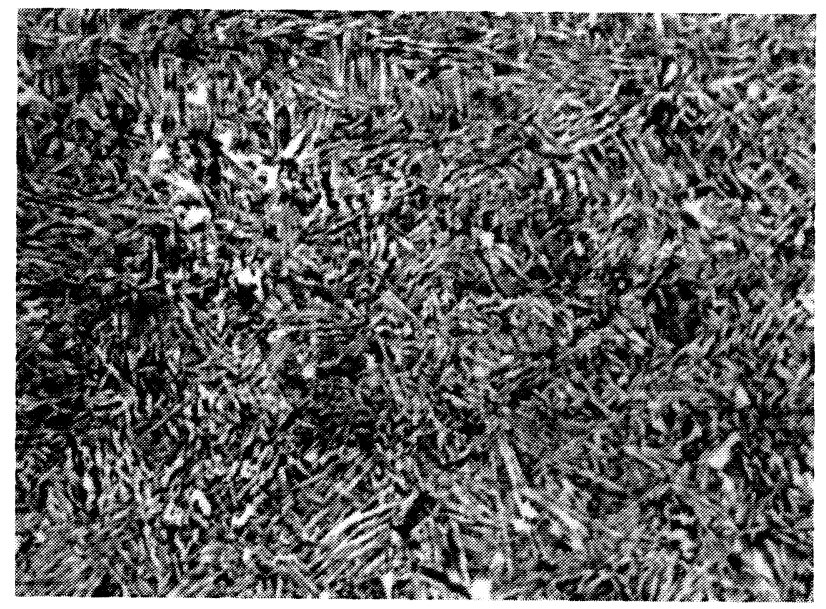

FIG. 1.- Estructura intermedia de temple; tubo núm. 1, diámetro $73 \mathrm{~mm}$, calidad C75.1. $\times 200$.

FIG. 1.- Intermediate quench structure; tube num. 1, diameter $73 \mathrm{~mm}$, quality C75.1. $\times 200$.
TABLA IV.- Limpieza de la tubería estudiada

TABLE IV.- Cleanness of the pipe material

\begin{tabular}{|c|c|c|c|}
\hline \multicolumn{2}{|c|}{ Tubo } & \multirow{2}{*}{ Estructura } & \multirow{2}{*}{ Figura } \\
\hline Núm. & $\begin{array}{c}\text { Diám., } \\
\text { mm }\end{array}$ & & \\
\hline $\begin{array}{l}1 \\
2\end{array}$ & $\begin{array}{l}73 \\
73\end{array}$ & $\begin{array}{l}\text { Gran cantidad de } \\
\text { escorias y silicatos } \\
\text { complejos gruesos } \\
\text { (núms. } 4 \text { - } 41 / 2 \text { de } \\
\text { la serie gruesa de ASTM) }\end{array}$ & $\begin{array}{l}5,6 \\
7 \text { y } 8\end{array}$ \\
\hline 3 & 60 & $\begin{array}{l}\text { El contenido de } \\
\text { inclusiones se considera } \\
\text { normal } \\
\text { Se evidencia alguna } \\
\text { escoria gruesa aislada }\end{array}$ & - \\
\hline 4 & 48 & $\begin{array}{l}\text { El acero se presenta } \\
\text { limpio, con un contenido } \\
\text { normal de microinclusiones }\end{array}$ & - \\
\hline
\end{tabular}

la dirección de laminación. El tubo núm. 2, además, presentó defectos de configuración en "festón" en su superficie exterior, localizados preferentemente en una de las generatrices de laminación.

La defectología transversal está compuesta por grietas cuasi perpendiculares a la superficie exterior y roturas subsuperficiales a ésta. La profundidad máxima observada fue de $0,6 \mathrm{~mm}$. Los bordes de grieta y las roturas se encuentran sin descarburar. Asimismo, en sección axial, se observan numerosas fisuras finas que, iniciándose en la superficie exterior, progresan de forma preferentemente intergranular a través del espesor; el camino de progresión se encuentra sin descarburar (Figs. 10, 11 y 12).

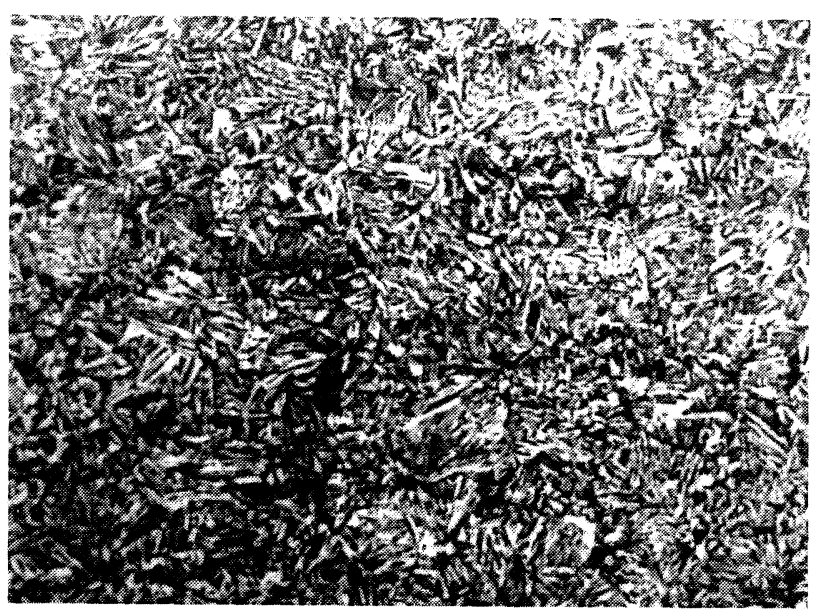

FIG. 2.- Estructura intermedia de temple; tubo núm. 2, diámetro $73 \mathrm{~mm}$, calidad C75.1. $\times 200$.

FIG. 2.- Intermediate quench structure; tube num. 1, diameter $73 \mathrm{~mm}$, quality C75.1. $\times 200$. 


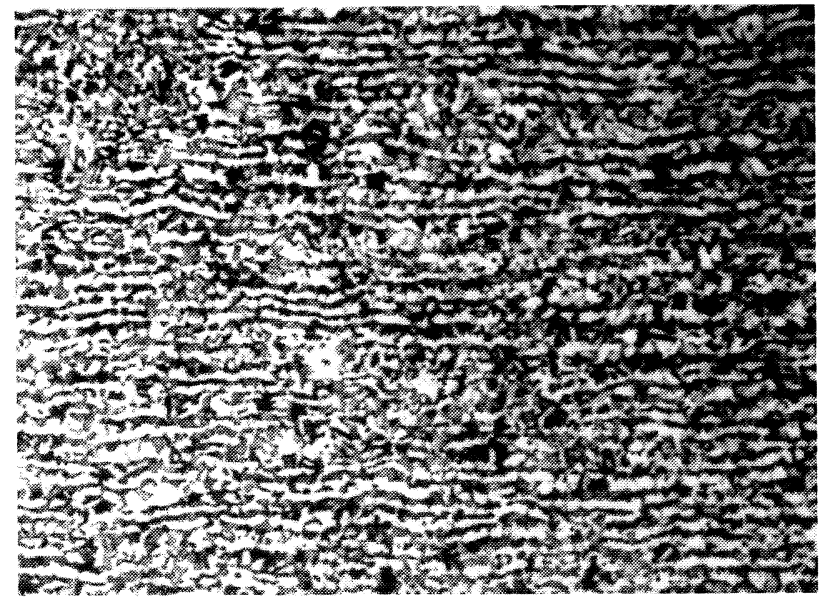

FIG. 3.- Estructura de ferrita y perlita; tubo núm. 3, diámetro60 mm, calidad J55. × 100 .

FIG. 3.- Ferrite + perlite structure, tube num. 3, diameter $60 \mathrm{~mm}$, quality J55. $\times 100$.

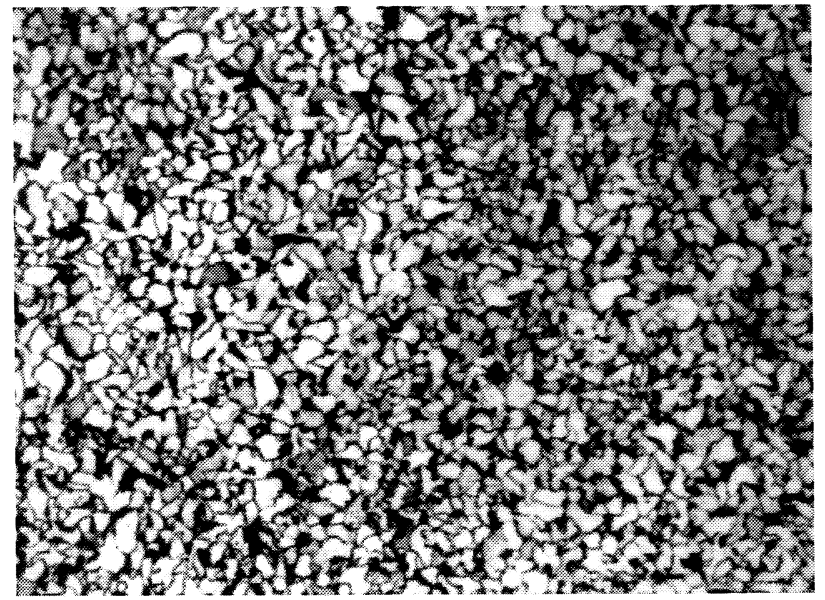

FIG. 4.- Estructura de ferrita y perlita; tubo núm. 4, diámetro $48 \mathrm{~mm}$, calidad Gr A. $\times 100$.

FIG. 4.- Ferrite + perlite structure, tube num. 4, diameter $48 \mathrm{~mm}$, quality $\mathrm{Gr}$ A. $\times 100$.

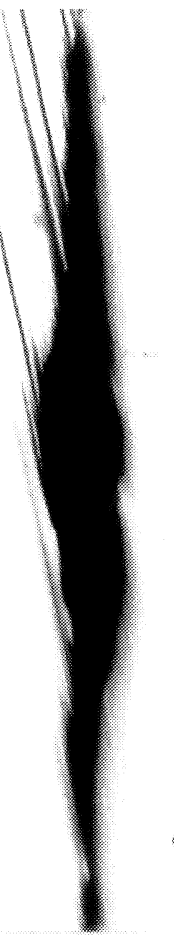

FIG. 5.

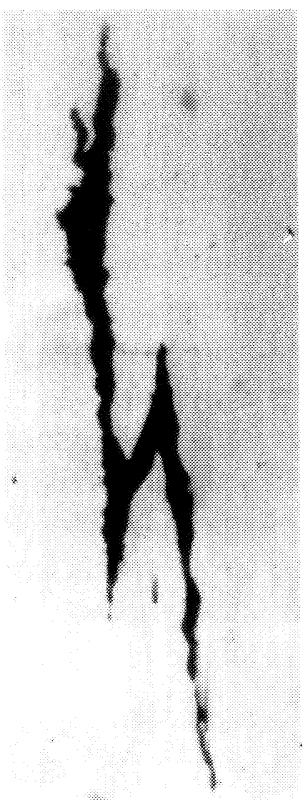

FIG. 6.

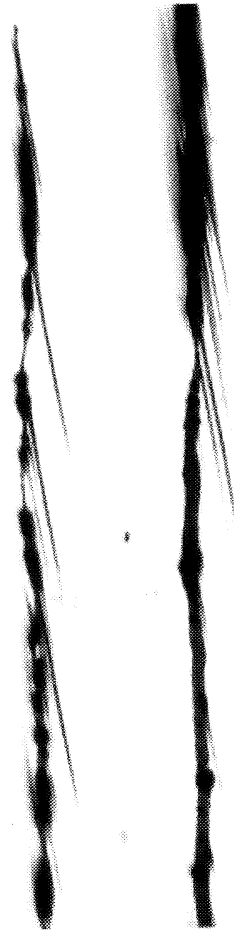

FIG. 7.

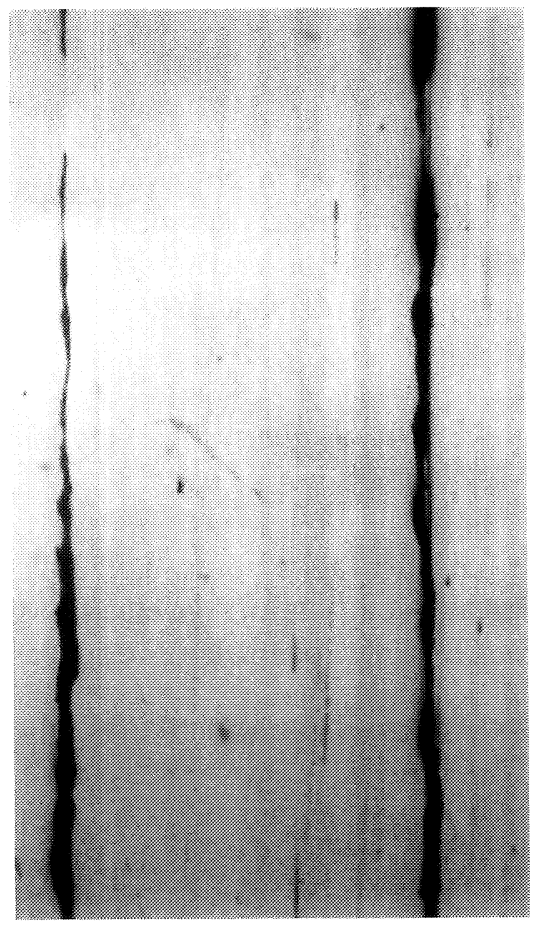

FIG. 8.

Fig. 5.- Escoria gruesa alargada; tubo núm. 1, diámetro $73 \mathrm{~mm} . \times 100$.

FIG. 5.-Long heavy slag; tube num. 1, diameter $73 \mathrm{~mm}$.

Fig. 6.- Segregación de escorias y de silicatos complejos; tubo núm. 2, diámetro $73 \mathrm{~mm} . \times 100$.

FIG. 6.- Segregation of slags and complex silicates; tube num. 2, diameter $73 \mathrm{~mm} . \times 100$.

Fig. 7.- Silicatos complejos gruesos orientados en el sentido de laminación; tubo núm. 1, diámetro $73 \mathrm{~mm}$. $\times 100$. FIG. 7.-Heavy complex silicates oriented in the rolling direction; tube num. 1, diameter $73 \mathrm{~mm} . \times 100$.

Fig. 8. - Silicatos complejos gruesos orientados en el sentido de laminación; tubo núm. 2, diámetro $73 \mathrm{~mm}$. $\times 100$. FIG. 8.-Heavy complex silicates oriented in the rolling direction; tube num. 2, diameter $73 \mathrm{~mm} . \times 100$. 


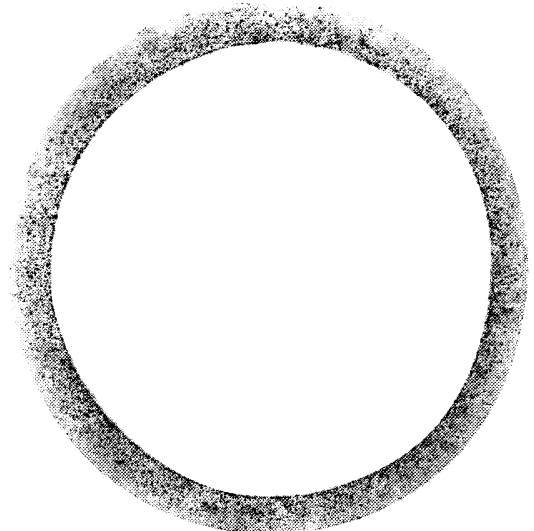

Tubo núm. 1
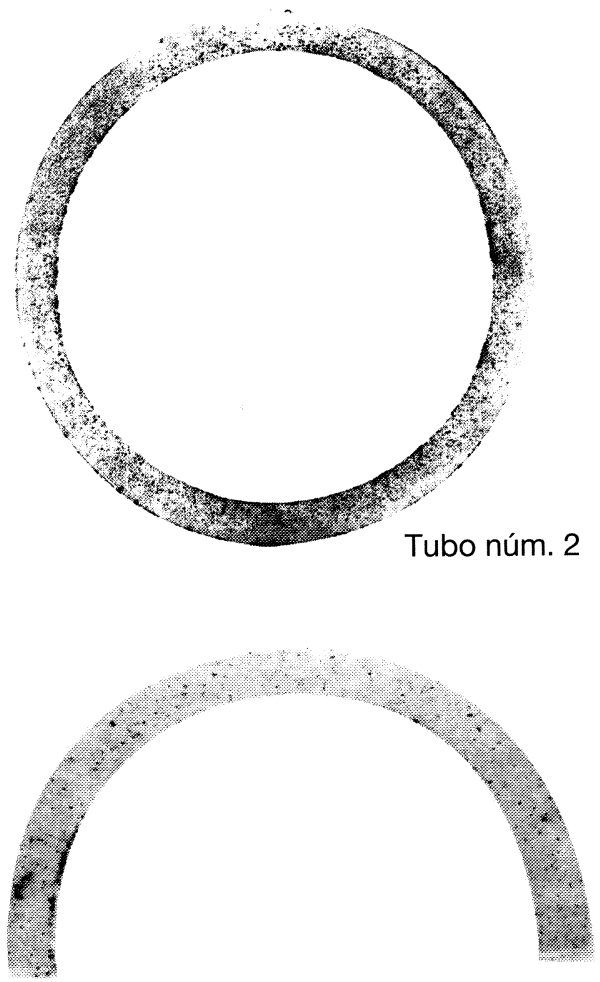

Tubo núm. 3

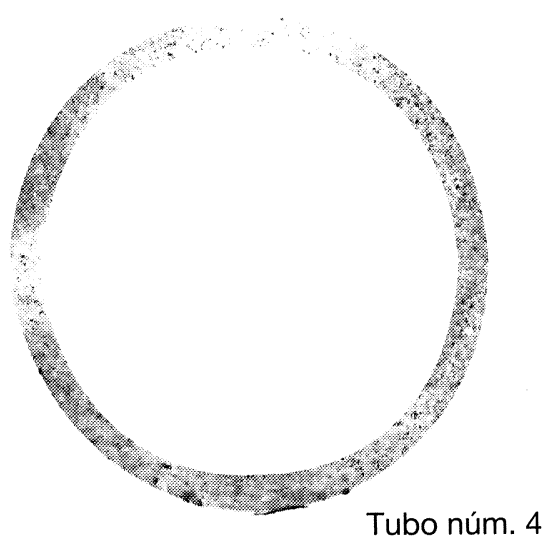

Fig. 9.- Impresiones Baumann en los cuatro tubos estudiados.

FIG. 9.-Baumann prints of the four tubes studied.

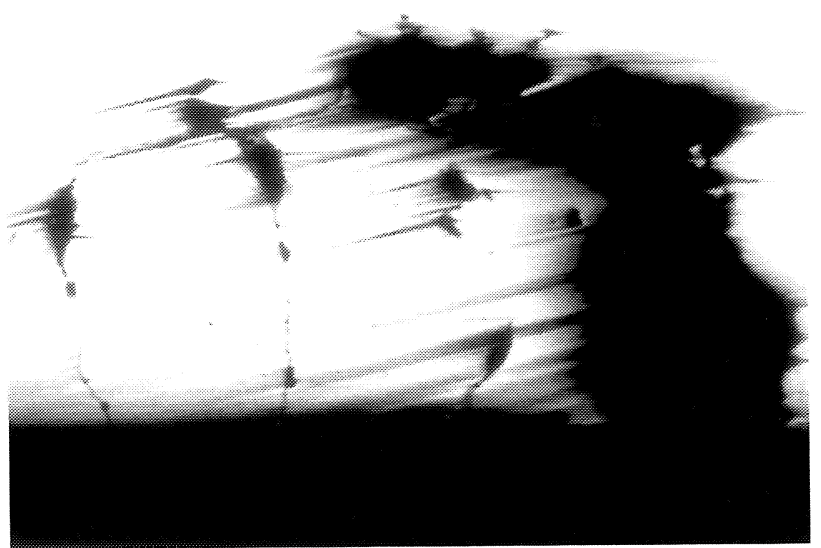

FIG. 10.- Agrietamiento superficial en los tubos núms. 1 y $2 . \times 75$.

FIG. 10.-Surface cracking in tubes 1 and $2 . \times 75$.

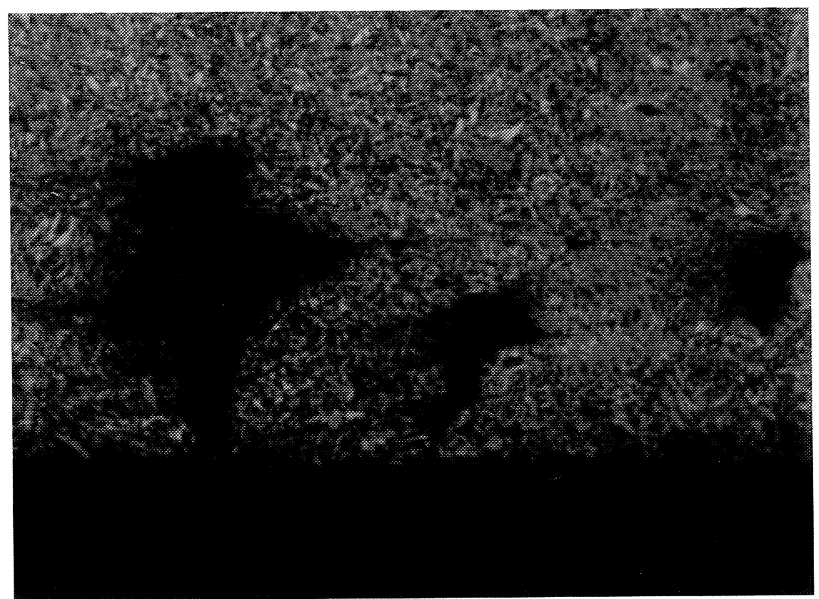

FIG. 11.- Roturas subsuperficial en bordes sin descarburación, en los tubos núms. 1 y 2. × 100 .

FIG. 11.- Subsurface failures in undecarburized edges in tubes 1 and $2 . \times 100$.

El defecto de configuración en "festón" está formado por un pliegue típico de laminación. Sus bordes están descarburados y presenta una profundidad máxima de 0,6 mm (Fig. 13).

\subsubsection{Tubo núm. 3: Calidad J55 y diámetro $60 \mathrm{~mm}$}

En el tubo núm. 3, la superficie interior presentaba protuberancias, laminaciones y estrías, todas ellas cortas, discontinuas, longitudinales y localizadas preferentemente en una franja de la generatriz de laminación.

Están originadas por escamación protuberante a la que se añaden oxidación superficial, agrietamiento superficial y pequeños desconchados. Se localizan, preferentemente, en minúsculas zonas carburadas subsuperficiales de la superficie interior (Figs. $14,15,16$ y 17). 




FIG. 12.- Fisuración con progresión intergranular en los tubos núms. 1 y $2 . \times 200$.

FIG. 12.- Intergranular fissuration in tubes 1 and $2 . \times 200$.

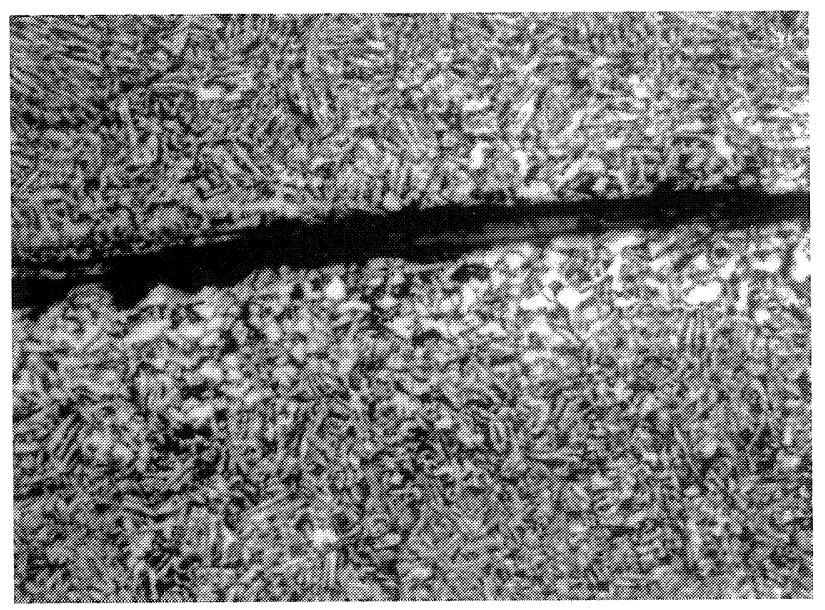

FIG. 13.- Pliegue con los bordes descarburados, correspondientes a los tubos núms. 1 y $2 . \times 200$.

FIG. 13.- Fold with decarburized edges correspondieng to tubes 1 and $2 . \times 200$.

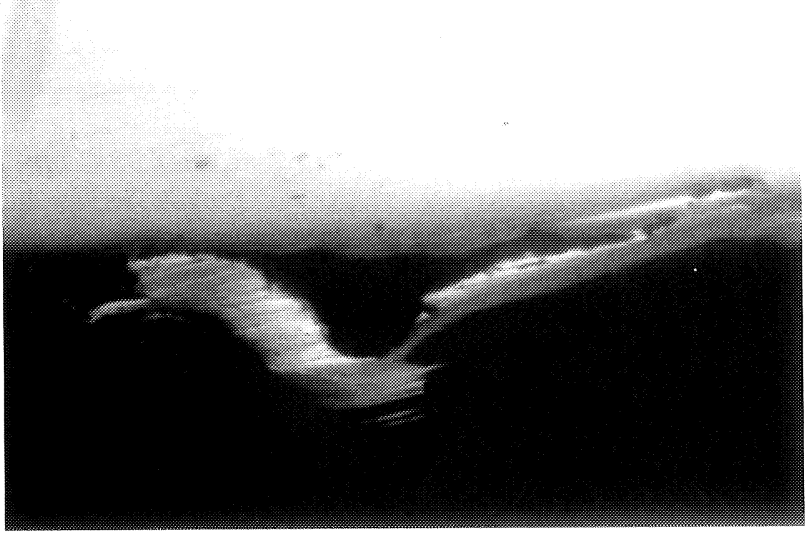

Fig. 14.-Desconchado en el tubo núm. 3. $\times 75$. FIG. 14.-Spalling in tube num. 3. $\times 75$.

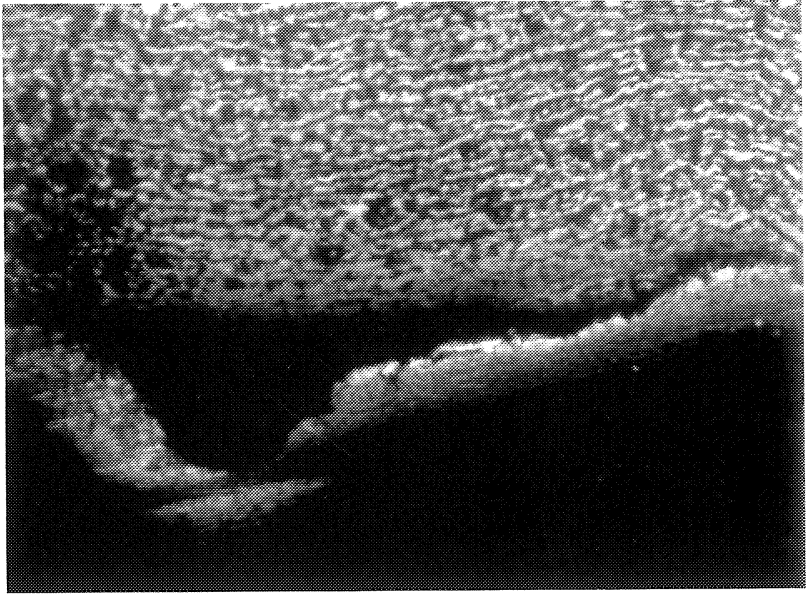

FIG. 15.- Desconchado con carburación subsuperficial en el tubo núm. 3. × 100 .

FIG. 15.- Spalling with subsurface carburization in tube num. 3. $\times 100$.

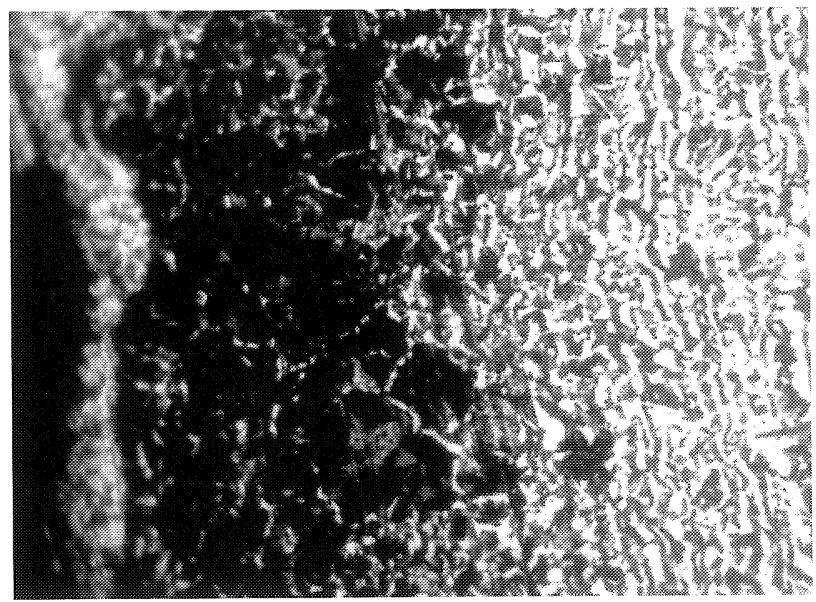

FIG. 16.- Carburación subsuperficial y capa superficial de óxidos en el tubo núm. 3. $\times 150$.

FIG. 16.- Subsurface carburization and oxide surface coat in tube $3 . \times 150$.

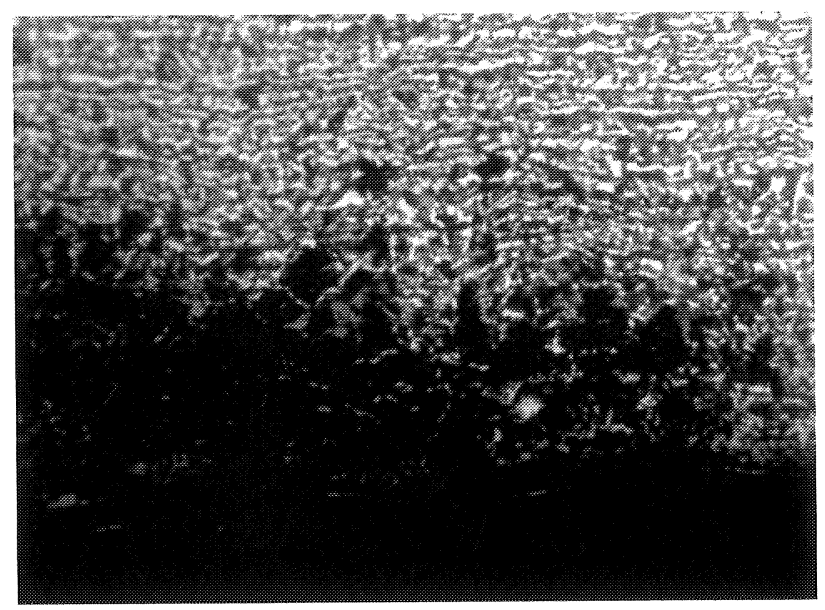

FIG. 17- Carburación subsuperficial en el tubo núm. 3. $\times 100$.

FIG. 17-Subsurface carburization in tube 3. $\times 100$. 


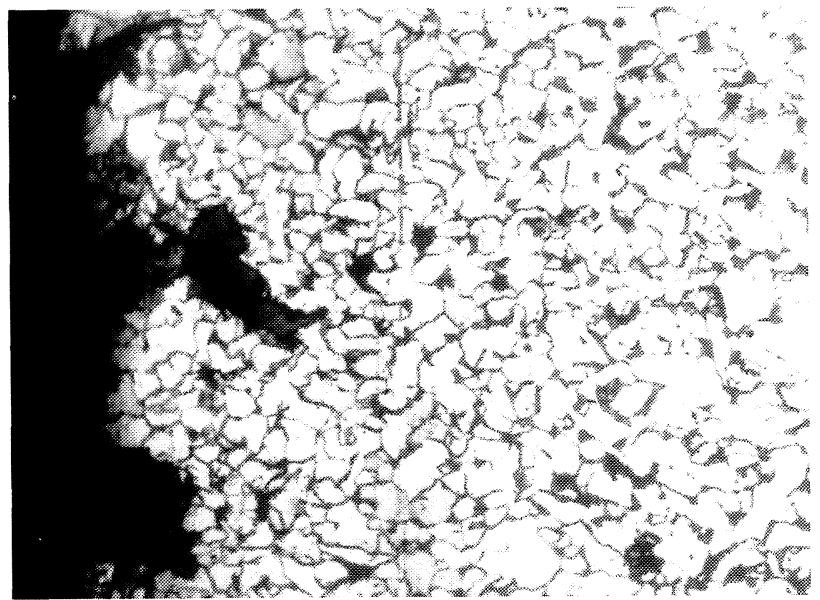

FIG. 18- Grietas y entallas oxidadas en el tubo núm. 4. $\times 150$.

FIG. 18-Oxidized cracks and notches in tube 4. $\times 150$.

\subsubsection{Tubo núm. 4: Calidad Gr A y diámetro $48 \mathrm{~mm}$}

En la superficie exterior del tubo núm. 4 se observa una defectología similar a la comentada para los tubos núms. 1 y 2 , pero aquí, los pequeños desgarres transversales presentan una profundidad máxima de $0,4 \mathrm{~mm}$. Estas fisuras se encuentran oxidadas y sus bordes sin descarburar (Figs. 18 y 19).

\section{ANÁLISIS DE RESULTADOS}

Según se ha visto, existen diversos defectos cuyo origen se trata a continuación:

- Pliegues: Se producen mecánicamente en los trenes de laminación.

- Inclusiones de escoria y de silicatos gruesos complejos: Proceden del lingote de partida, y se alargan y orientan en el sentido de la laminación.

- Grietas y roturas superficiales: Son de origen mecánico, produciéndose en los trenes durante la laminación. Por anomalías durante el proceso, se ocasionan zonas microtensionalmente altas que inducen un elevado potencial vector que distorsiona localmente la estructura, dando lugar a los típicos desgarres.

- Escamación y desconchado: En el caso que nos ocupa (tubo núm. 3) son de origen metalúrgico. Existen puntos locales duros de matriz perlítica como consecuencia de anomalías en el calentamiento (alto potencial carburante y oxidante de la atmósfera del horno) que en la laminación,

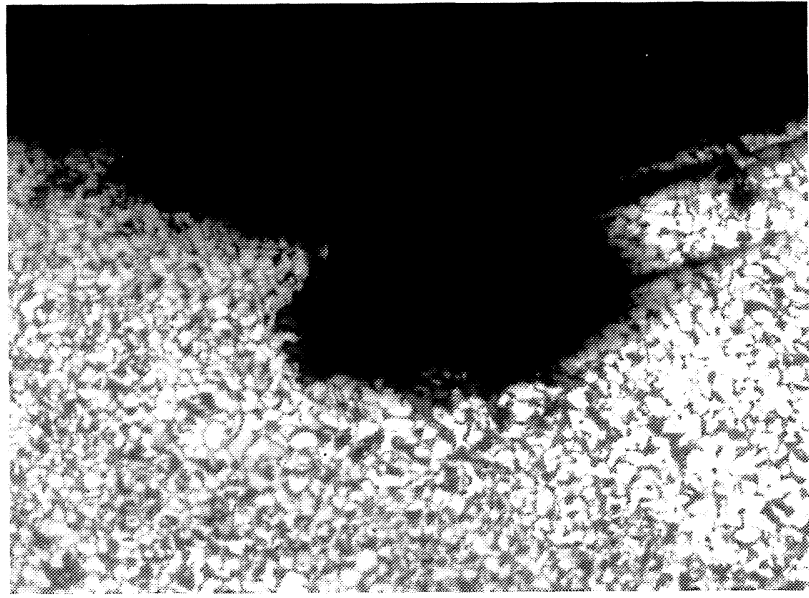

FIG. 19.- Rotura superficial con los bordes sin descarburar en el tubo núm. 4. $\times 75$.

FIG. 19.- Surface failure with undecarburized edges in tube $4 . \times 75$.

por tener una elevada dureza, se escaman y desconchan.

\section{CONCLUSIONES}

La tubería estudiada presenta los defectos de fabricación siguientes:

- Inclusiones de escoria y de silicatos complejos gruesos, formados en el lingote de partida.

- Pliegues, de origen mecánico, producidos en los trenes de laminación.

- Desgarres, grietas y roturas superficiales, que tienen su origen en el proceso mecánico-metalúrgico y se producen en los trenes de laminación.

- Escamación y desconchado, que en el caso estudiado es de origen metalúrgico.

\section{REFERENCIAS}

(1) American Petroleum Institute (API). Specification for Casing and Tubing. Washington. Spec. 5CT. Thirty-ninth edition. June 1, 1991.

(2) American Petroleum Institute (API). Specification for Line Pipe. Washington. Spec. 5L. Thirty-ninth edition. June 1, 1991.

(3) THIELSCH, H. Defectos y roturas en recipientes a presión y tuberías. Ed. Urmo, S.A. Bilbao. 1981: 117-149.

(4) Karl Naumann, F. y Spies, F. Case histories in failure analysis. Max Planck Institut für Eisenforschung. Düsseldorf. American Society for Metals. ASM. 1985: 45-48, 73 $75,123-128$ y $309-318$.

(5) Hutchings, F.R. y UnTERweiser, P.M. Failure Analysis: The British Engine Technical Reports. American Society for Metals. ASM. Metals Park. Ohio, 1981. 